\title{
Análise comparativa das coordenadas no Sistema Geodésico Local e no Sistema Topográfico Local
}

\author{
Débora Paula Simões \\ deborapaula.ds@gmail.com \\ orcid.org/0000-0002-1615-988X \\ Instituto Federal de Educação, Ciência e \\ Tecnologia do Sul de Minas Gerais \\ (IFSULDEMINAS) - Campus \\ Inconfidentes, Inconfidentes, Minas \\ Gerais, Brasil. \\ Fábio Luiz Albarici \\ fabio.albarici@ifsuldeminas.edu.br \\ orcid.org/0000-0003-4436-0561 \\ Instituto Federal de Educação, Ciência e \\ Tecnologia do Sul de Minas Gerais \\ (IFSULDEMINAS) - Campus \\ Inconfidentes, Inconfidentes, Minas \\ Gerais, Brasil. \\ Paulo Augusto Ferreira Borges \\ paulo.borges@ifsuldeminas.edu.br \\ orcid.org/0000-0002-4792-8637 \\ $\frac{\text { orcid.org/0000-0002-4792-8637 }}{\text { Instituto Federal de Educação, Ciência e }}$ \\ Tecnologia do Sul de Minas Gerais \\ Tecnologia do Sul de Minas G
(IFSULDEMINAS) - Campus \\ Inconfidentes, Inconfidentes, Minas \\ Gerais, Brasil.
}

\begin{abstract}
RESUMO
Uma das atividades importantes dos profissionais em Geodésia consiste nas transformações entre sistemas de coordenadas e, dependendo da finalidade, deve-se recorrer a um ou outro sistema. Os sistemas de coordenadas locais possibilitam maior precisão na implantação de obras de engenharia e nos trabalhos cadastrais, dentre os quais destaca-se o Sistema Topográfico Local, adotado em levantamentos municipais para fins de cadastro, conforme a NBR 14166/98, e o Sistema Geodésico Local, aplicado quando no georreferenciamento de imóveis rurais, de acordo com o Manual Técnico de Posicionamento da NTGIR (INCRA, 2013a). Com o objetivo de realizar uma análise comparativa das coordenadas obtidas nos dois sistemas de coordenadas tridimensionais locais, adotou-se, como objeto de estudo, vinte e oito marcos localizados no IFSULDEMINAS e uma estação geodésica homologada pelo IBGE, os quais tiveram suas coordenadas geodésicas obtidas por meio do posicionamento por GNSS, com os métodos relativo estático e estático rápido. Conclui-se, a partir das coordenadas locais obtidas e das comparações pontuais, lineares e de superfície realizadas, que ambos os sistemas apresentam uma divergência pequena, contudo significativa, o que faz com que os mesmos não sejam considerados equivalentes e, desse modo, a aplicabilidade de cada um deve obedecer às exigências das normas técnicas.
\end{abstract}

PALAVRAS-CHAVE: GNSS. Sistema Geodésico. Sistema Topográfico. Coordenadas locais. 


\section{INTRODUÇÃO}

Uma das atividades de muita importância do profissional em agrimensura e cartografia consiste nas transformações entre sistemas de coordenadas, o que pode ser entendido como a relação das coordenadas de um ponto da superfície terrestre em dois sistemas de coordenadas diferentes. Dependendo da finalidade e do trabalho a ser realizado, é necessário que se recorra a um ou outro sistema específico. É comum, em projetos de engenharia e cadastros, a utilização de um sistema de coordenadas locais, o qual possibilita maior precisão na implantação de obras e a geração de plantas cadastrais com uma melhor qualidade. Segundo Monico (2008), os sistemas de coordenadas terrestres locais proporcionam apoio para levantamentos tridimensionais locais. Dentre eles destaca-se o Sistema Geodésico Local e o Sistema Topográfico Local.

O Sistema Geodésico Local é adotado nos trabalhos de georreferenciamento de imóveis rurais e, segundo o Manual Técnico de Posicionamento (INCRA, 2013a), pode ser aplicado no cálculo de áreas, no uso do método de posicionamento por geometria analítica e em projetos de parcelamento/desmembramento. Além disso, de acordo com Monico (2008), no posicionamento por satélite, o Sistema Geodésico Local também apresenta grande utilidade, sendo usado para obter o azimute e o ângulo vertical e para analisar, em termos de componentes horizontal e vertical, o erro em cada uma das coordenadas $X, Y$ e Z. Já o Sistema Topográfico Local, segundo a NBR 14166/98, consiste no sistema de representação, em planta, das posições relativas de pontos de um levantamento topográfico com origem em um ponto de coordenadas geodésicas conhecidas (ABNT, 1998). No Brasil, o Sistema Topográfico Local é adotado em levantamentos municipais para fins de cadastro.

Diante das diversas aplicabilidades referentes aos sistemas de coordenadas locais, objetiva-se por meio desse estudo realizar uma análise comparativa das coordenadas no Sistema Topográfico Local e no Sistema Geodésico Local, evidenciando-se as discrepâncias existentes entre ambos os sistemas por meio de comparações envolvendo grandezas pontuais, lineares e de superfície.

\section{FUNDAMENTAÇÃO TEÓRICA}

\section{POSICIONAMENTO POR GNSS NOS MÉTODOS RELATIVO ESTÁTICO E RELATIVO ESTÁTICO RÁPIDO}

O posicionamento relativo tem por objetivo a determinação do vetor entre dois pontos, chamado de vetor da linha de base ou simplesmente linha de base (HOFMANN-WELLENHOF et al, 2008). Segundo o Manual Técnico de Posicionamento, da Norma Técnica de Georreferenciamento de Imóveis Rurais (NTGIR), 3a edição, no posicionamento relativo é necessário que dois ou mais receptores GNSS coletem dados simultaneamente, onde ao menos um dos receptores ocupe um vértice de referência. Ainda de acordo com o manual, no posicionamento relativo podem ser utilizadas as seguintes observáveis: fase da onda portadora, pseudodistância ou as duas em conjunto; sendo que a fase da onda portadora proporciona a melhor precisão (INCRA, 2013a). 
No posicionamento relativo estático, dois ou mais receptores rastreiam simultaneamente, os satélites visíveis por um período de tempo que pode variar de dezenas de minutos (20 minutos, no mínimo) até algumas horas. Já no posicionamento relativo estático rápido, um receptor serve como base, permanecendo fixo sobre uma estação de referência, coletando dados, enquanto outro percorre as estações de interesse (receptor móvel), em cada uma das quais permanece parado cerca de 5 a 20 minutos para coletar dados. Nesse último método, o vetor das ambiguidades envolvido em cada linha-base deve ser solucionado, isto é, fixado como inteiro, para que os resultados obtidos apresentam razoável nível de precisão (MONICO, 2008).

\section{SISTEMA GEODÉSICO LOCAL}

De acordo com a NTGIR, 3a edição, o Sistema Geodésico Local (SGL) é um sistema cartesiano composto de três eixos mutuamente ortogonais $(e, n, u)$, onde o eixo " $\mathrm{n}$ " aponta em direção ao norte geodésico, o eixo " $\mathrm{e}$ " aponta para a direção leste e é perpendicular ao eixo " $\mathrm{n}$ ", ambos contidos no plano topocêntrico, e o eixo " $\mathrm{u}$ " coincide com a normal ao elipsoide que passa pelo vértice escolhido como a origem do sistema (INCRA, 2013b). A Figura 1 ilustra um ponto sobre a superfície terrestre associado ao Sistema Geodésico Local e ao Sistema Geocêntrico.

Figura 1 - Sistema Geodésico Local e Sistema Geocêntrico

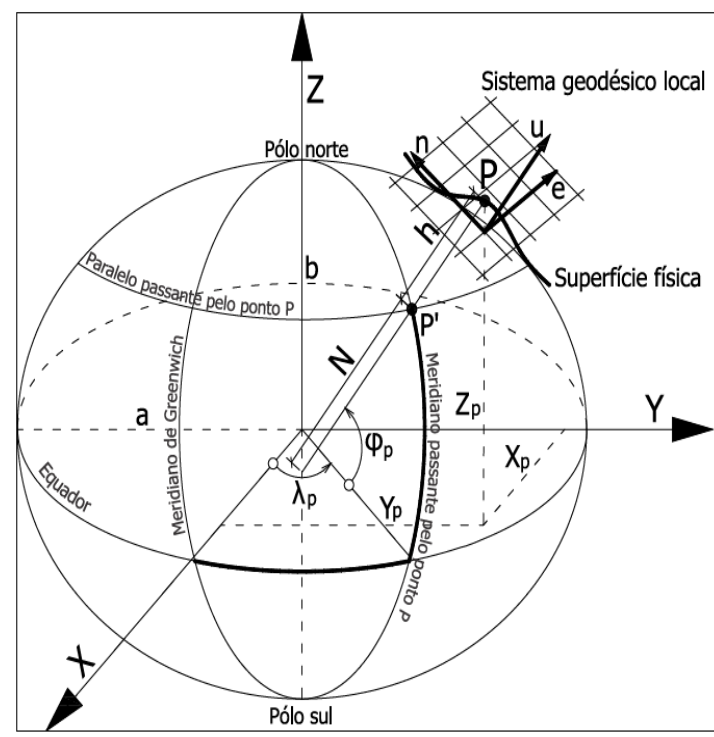

Fonte: INCRA (2013a).

Segundo a NTGIR, 3ạ edição, as coordenadas cartesianas geocêntricas, correspondentes ao Sistema Geocêntrico, são coordenadas referenciadas a três eixos ortogonais com origem no centro de massa da terra, sendo o eixo Z orientado na direção do Conventional Terrestrial Pole (Pólo Terrestre Convencional), o eixo $X$ na direção média do meridiano de Greenwich e o eixo $Y$ de modo a tornar o sistema dextrógiro (INCRA, 2013b). Apresenta-se a seguir (Equações 1 a 5) a formulação matemática com a qual se obtêm as coordenadas geocêntricas $\left(X_{c}, Y_{c}, Z_{c}\right)$ de um ponto cujas coordenadas geodésicas $(\varphi, \lambda$, h) são conhecidas (RAPP, 1991). 


$$
\begin{aligned}
X_{c} & =(N+h) \cos \varphi \cos \lambda \\
Y_{c} & =(N+h) \cos \varphi \sin \lambda \\
Z_{c} & =\left[N\left(1-e^{2}\right)+h\right] \sin \varphi \\
N & =\frac{a}{\left(1-e^{2} \sin ^{2} \varphi\right)^{1 / 2}} \\
e^{2} & =\frac{a^{2}-b^{2}}{a^{2}}
\end{aligned}
$$

Em que:

$X_{c}, Y_{c}, Z_{c}=$ correspondem às coordenadas geocêntricas de um ponto com coordenadas geodésicas conhecidas, o qual se deseja determinar suas coordenadas cartesianas locais (e, n e u);

$\varphi, \lambda, h=$ coordenadas geodésicas (latitude, longitude e altura geométrica) conhecidas do referido vértice;

$N=$ raio de curvatura da seção primeiro vertical;

$e^{2}=$ quadrado da primeira excentricidade (e) do elipsoide de referência, sendo a e b os semi-eixos maior e menor, respectivamente, do elipsoide de referência adotado no SGB (Sistema Geodésico Brasileiro).

Dispondo-se das coordenadas geocêntricas $\left(X_{c}, Y_{c}, Z_{c}\right)$ de um determinado vértice é possível obter as coordenadas cartesianas locais $(e, n, u)$, que correspondem a coordenadas cartesianas definidas num plano topocêntrico local perpendicular à normal e tangente ao elipsoide elevado à superfície terrestre no ponto origem do SGL (INCRA, 2013b), adotando-se o método das rotações e translações (INCRA, 2013a), conforme modelo funcional dado pela Equação 6.

$$
\left[\begin{array}{l}
e \\
n \\
u
\end{array}\right]=\left[\begin{array}{ccc}
1 & 0 & 0 \\
0 & \sin \varphi_{0} & \cos \varphi_{0} \\
0 & -\cos \varphi_{0} & \sin \varphi_{0}
\end{array}\right]\left[\begin{array}{ccc}
-\sin \lambda_{0} & \cos \lambda_{0} & 0 \\
-\cos \lambda_{0} & -\sin \lambda_{0} & 0 \\
0 & 0 & 1
\end{array}\right] \cdot\left[\begin{array}{l}
X-X_{0} \\
Y-Y_{0} \\
Z-Z_{0}
\end{array}\right]+\left[\begin{array}{l}
e_{0} \\
n_{0} \\
u_{0}
\end{array}\right]
$$

Em que:

$e, n, u=$ coordenadas cartesianas locais do vértice de interesse;

$e_{0}, n_{0}, u_{0}=$ coordenadas arbitradas para o vértice origem para que as coordenadas cartesianas locais de interesse não sejam negativas;

$X_{c}, Y_{c}, Z_{c}=$ coordenadas cartesianas geocêntricas do vértice de interesse;

$\varphi_{0}, \lambda_{0}=$ latitude e longitude adotadas como origem do sistema;

$X_{0}, Y_{0}, Z_{0}=$ coordenadas cartesianas geocêntricas adotadas como origem do sistema.

Página | 65

\section{SISTEMA TOPOGRÁFICO LOCAL}


O Sistema Topográfico Local (STL), segundo a NBR 13133/94, consiste no sistema de projeção utilizado nos levantamentos topográficos pelo método direto clássico para a representação das posições relativas dos acidentes levantados, através de medições angulares e lineares, horizontais e verticais.

O plano de representação, origem, eixos e orientação são os elementos constituintes do sistema, fundamentais para o posicionamento dos pontos do levantamento por intermédio de um sistema cartesiano ortogonal em duas dimensões, em que os eixos $X$, positivo no sentido leste, e $Y$, coincidente à linha meridiana geográfica, estão jacentes no Plano do Horizonte Local, adotando-se, para efeito de cálculos, a esfera de adaptação de Gauss como figura geométrica da terra. $\mathrm{O}$ plano do horizonte local é elevado à altitude ortométrica, $\mathrm{H}$, média da área de abrangência do sistema, passando a chamar-se Plano Topográfico Local, conforme indicado na Figura 2 (ABNT, 1998).

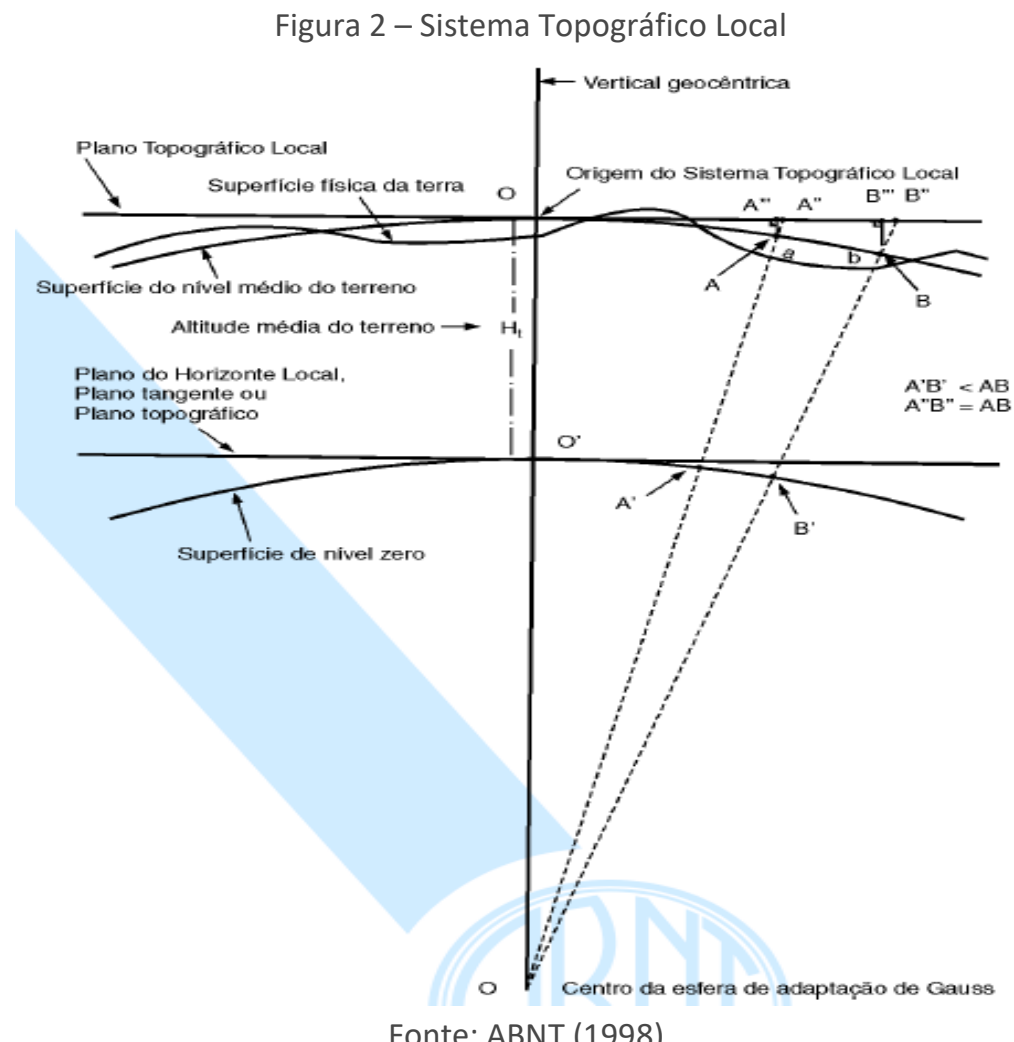

As coordenadas plano-retangulares $(\mathrm{X}, \mathrm{Y})$ dos marcos geodésicos de apoio imediato no Sistema Topográfico Local são obtidas a partir de suas coordenadas geodésicas $(\varphi, \lambda)$ e das coordenadas geodésicas da origem do sistema $\left(\varphi_{0}, \lambda_{0}\right)$. Às coordenadas plano-retangulares da origem do Sistema Topográfico Local $(0,0)$ são adicionados os termos constantes $150000,000 \mathrm{~m}$ e $250000,000 \mathrm{~m}$, respectivamente, para a abscissa (X) e para a ordenada (Y), no escopo de evitarem-se valores negativos nos demais pontos da área de abrangência do sistema (ABNT, 1998). A partir das coordenadas plano-retangulares dos pontos geodésicos de apoio imediato são obtidas as coordenadas plano-retangulares dos pontos levantados, topograficamente, na área de abrangência do Sistema Topográfico Local (ABNT, 1998), de acordo com as equações apresentadas a seguir (Equação 7 a Equação 22). 


$$
\begin{aligned}
& X=150000+x_{p} \\
& Y=250000+y_{p} \\
& x_{p}=-\left(\Delta \lambda_{1} \cos \varphi_{p} N_{p} \operatorname{arc1} 1^{\prime \prime}\right) \cdot c \\
& y_{p}=\frac{1}{B}\left[\Delta \varphi_{1}+C x_{p}{ }^{2}+D\left(\Delta \varphi_{1}\right)^{2}+E\left(\Delta \varphi_{1}\right) x_{p}{ }^{2}+E C x_{p}{ }^{4}\right] \cdot c \\
& \Delta \lambda=\lambda_{p}-\lambda_{o} \\
& \Delta \varphi=\varphi_{p}-\varphi_{o} \\
& \Delta \lambda_{1}=\Delta \lambda^{\prime \prime}\left[1-3,9173.10^{-12}\left(\Delta \lambda^{\prime \prime}\right)^{2}\right] \\
& \Delta \varphi_{1}=\Delta \varphi^{\prime \prime}\left[1-3,9173 \cdot 10^{-12}(\Delta \varphi ")^{2}\right] \\
& B=\frac{1}{M_{0} \operatorname{arc1"}} \\
& C=\frac{\tan \varphi_{0}}{2 M_{0} N_{0} \operatorname{arc1} 1 "} \\
& D=\frac{3 e^{2} \sin \varphi_{0} \cos \varphi_{0} \operatorname{arc1} 1 "}{2\left(1-e^{2} \sin ^{2} \varphi_{0}\right)} \\
& E=\frac{1+3 \tan \varphi_{0}}{6 N_{0}^{2}} \\
& c=\frac{R_{o}+H}{R_{0}} \\
& R_{0}=\sqrt{M_{0} N_{0}} \\
& M_{0}=\frac{a\left(1-e^{2}\right)}{\left(1-e^{2} \sin ^{2} \varphi_{0}\right)^{3 / 2}} \\
& N_{0}=\frac{a}{\left(1-e^{2} \sin ^{2} \varphi_{0}\right)^{1 / 2}}
\end{aligned}
$$

\section{Em que:}

$X, Y=$ coordenadas plano-retangulares no Sistema Topográfico Local;

$\varphi_{p}, \lambda_{p}=$ coordenadas geodésicas (latitude e longitude, respectivamente) do vértice $\mathrm{P}$ considerado;

$\varphi_{0}, \lambda_{0}=$ coordenadas geodésicas do ponto origem do sistema;

$M_{0}=$ raio de curvatura da seção meridiana do elipsoide de referência em $\mathrm{P}_{0}$ (origem do sistema);

$N_{0}=$ raio de curvatura da seção normal ao plano meridiano do elipsoide de referência em $\mathrm{P}_{0}$;

$$
c=\text { fator de elevação; }
$$



local.

$H=$ altitude ortométrica média do terreno ou altitude do plano topográfico

Conforme Monico (2008), a altitude ortométrica $(H)$ é vinculada ao campo de gravidade da Terra e tem valor aproximado obtido por meio da Equação 23, na qual $h$ corresponde à altitude geométrica, proporcionada pelo GNSS, e $G$ corresponde à ondulação do geoide.

$$
\mathrm{H} \cong \mathrm{h}-\mathrm{G}
$$

Segundo a NBR 14166, as coordenadas plano-retangulares no Sistema Topográfico Local subentendem que as observações angulares, horizontais e lineares, nas operações topográficas de sua determinação, estão representadas em verdadeira grandeza no plano topográfico local, com orientação, em planta, para o norte da quadrícula, o que significa que estão afetadas pela convergência meridiana, a qual só é nula para pontos situados ao longo do meridiano da origem do sistema, e somente o eixo das ordenadas $(\mathrm{Y})$ está orientado para o norte geográfico (ABNT, 1998). Para cálculo da convergência meridiana adotamse as seguintes equações (Equações 24 a 27):

$$
\begin{aligned}
& \gamma_{p}=-\left[\Delta \lambda^{\prime \prime} \sin \varphi_{m} \sec \frac{\Delta \varphi}{2}+F\left(\Delta \lambda^{\prime \prime}\right)^{3}\right] \\
& F=\frac{\sin \varphi_{m} \cos \varphi_{m} \sin ^{2} 1^{\prime \prime}}{12} \\
& \Delta \lambda^{\prime \prime}=\left(\lambda_{p}-\lambda_{0}\right) \cdot 3600 \\
& \Delta \varphi=\varphi_{p}-\varphi_{0}
\end{aligned}
$$

Sendo:

$\gamma_{p}=$ convergência meridiana no ponto considerado, que corresponde à diferença entre o azimute direto na direção $\mathrm{OP}(\mathrm{O}$ - origem do sistema e $\mathrm{P}$ ponto geodésico considerado) e o azimute recíproco desta direção (PO) (ABNT, 1998);

$\varphi_{m}=$ latitude média entre o ponto geodésico considerado (P) e a origem do sistema (O)

Quanto à área de abrangência do Sistema Topográfico Local, a NBR 14166/98 salienta que as coordenadas plano-retangulares devem estar limitadas, em seus valores absolutos (isentas dos termos constantes), ao máximo de $50000 \mathrm{~m}$, de maneira que nenhum ponto diste da origem do sistema mais que $70710,68 \mathrm{~m}$, o que garantirá um erro relativo decorrente da desconsideração da curvatura terrestre menor que 1:50000 nesta dimensão e 1:20000 nas imediações de sua extremidade (ABNT, 1998). 


\section{METODOLOGIA}

Para realizar a comparação entre coordenadas cartesianas locais, referidas ao Sistema Geodésico Local, e coordenadas topográficas locais, referidas ao Sistema Topográfico Local, rastreou-se vinte e oito vértices materializados por concreto, localizados ao longo do perímetro da Fazenda Escola, pertencente ao IFSULDEMINAS - Campus Inconfidentes. Na Figura 3 destaca tais vértices, nomeando-os com o prefixo M, cuja numeração varia de 01 a 28.

Figura 3 - Localização dos vinte e oito marcos na Fazenda Escola do IFSULDEMINAS Campus Inconfidentes

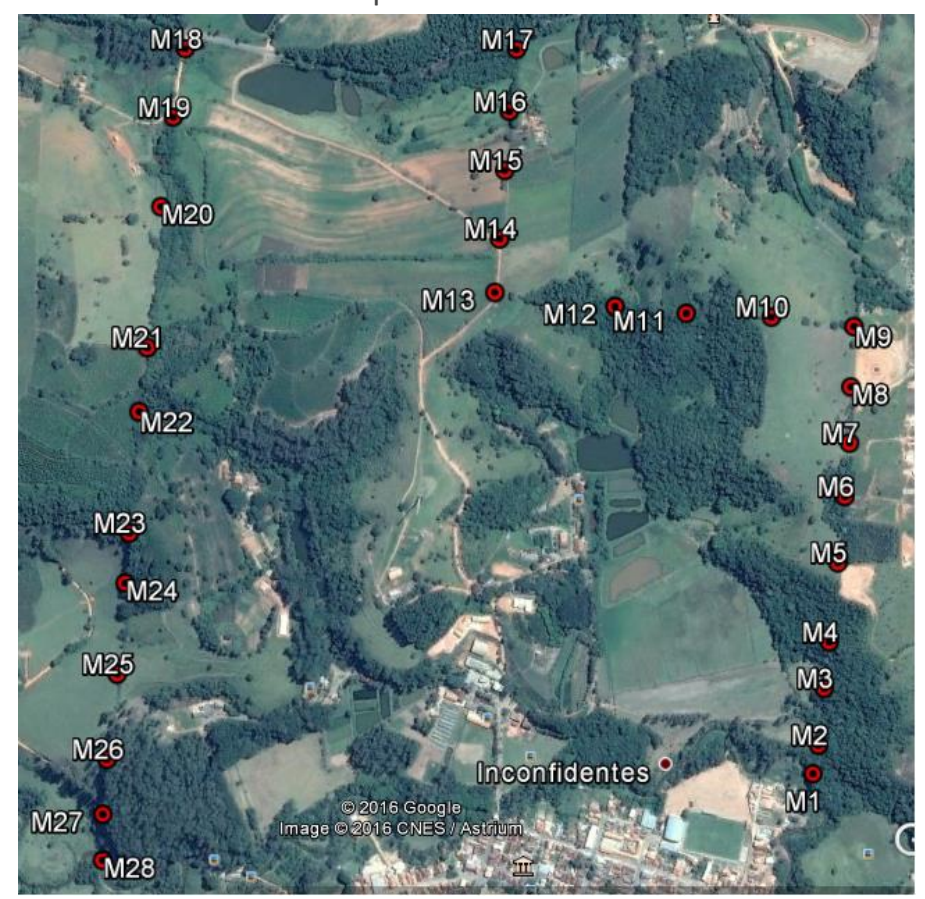

Fonte: Google Earth (2016).

Objetivando-se determinar com precisão as coordenadas geodésicas $(\varphi, \lambda, h)$ desses marcos, realizou-se o posicionamento por GNSS, adotando-se os métodos relativo estático e estático rápido. Para isso, utilizou-se de receptores GNSS da marca Spectra Precision, modelo ProMarkTM 220, bem como suas respectivas antenas GNSS (modelo ASH 111661). Dispondo-se de tais equipamentos, adotouse um tempo de rastreio entre 10 e 30 minutos em cada marco rastreado, levando em consideração as condições externas do local para determinar o tempo suficiente de rastreio que garantisse a obtenção de coordenadas precisas, adotando-se, assim, ora o método estático ora o estático rápido. Já as coordenadas da estação geodésica (Estação 93949), utilizada como ponto de controle no processamento dos dados levantados e ilustrada pela Figura 4, foram obtidas por meio de seu respectivo relatório disponível no site do IBGE, no seguinte endereço eletrônico: http://www.ibge.gov.br/home/geociencias/ geodesia/bdgpesq_googlemaps.php\#tabela_dados. 
Figura 4 - Estação 93949, IFSULDEMINAS - Campus Inconfidentes

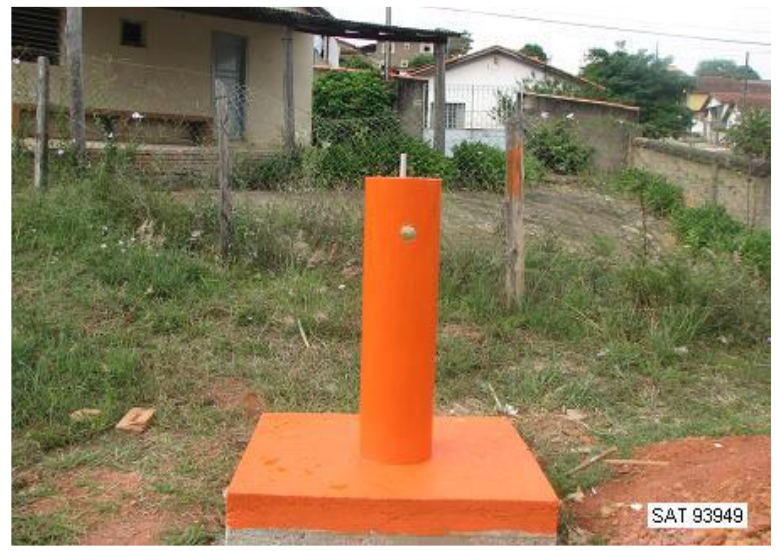

Fonte: IBGE (2016).

Dispondo-se dos arquivos brutos, obtidos no posicionamento por GNSS, processou-se os dados coletados através do software GNSS Solutions ${ }^{T M}$ v3.80.8. Tanto no rastreio dos marcos, como no processamento dos dados, utilizou-se o sistema de referência SIRGAS 2000 (Sistema de Referência Geocêntrico para as Américas, em sua realização no ano 2000), no fuso $23 \mathrm{~S}$ e meridiano central de$45^{\circ}$, vinculando-se as coordenadas ao SGB e obtendo-se as coordenadas geodésicas $(\varphi, \lambda, h)$ e na projeção UTM $(E, N, h)$ dos vértices de estudo.

Aplicou-se, então, a transformação de coordenadas geodésicas em coordenadas cartesianas geocêntricas, utilizando-se as expressões apresentadas nas Equações 1 a 5 . A partir dessas últimas, determinou-se as coordenadas cartesianas locais $(e, n, u)$ de cada um dos marcos e da estação geodésica 93949, adotando-se a expressão matricial apresentada pela Equação 6. Em seguida, realizou-se o cálculo das coordenadas topográficas locais $(X, Y, Z)$ utilizando-se o software DataGeosis Office v7.5.10.3, o qual adota as equações apresentadas na seção 2.3.

Como o STL constitui-se num sistema de coordenadas planas $(\mathrm{X}, \mathrm{Y})$, conforme a NBR 14166/98, assumiu-se que as coordenadas referentes ao eixo $z$ equivalem às suas respectivas altitudes ortométricas, as quais foram calculadas utilizando-se o software MAPGEO 2015 v1.0. Obtendo-se a ondulação geoidal para cada ponto no MAPGEO 2015 v1.0, aplicou-se a Equação 23 e determinou-se as altitudes ortométricas, sendo as altitudes geométricas conhecidas a partir do processamento dos dados obtidos no posicionamento por GNSS.

Dispondo-se das coordenadas topográficas locais e das coordenadas cartesianas locais dos marcos e da estação geodésica, realizou-se uma análise das mesmas, a fim de se comprovar a compatibilidade dos sistemas em estudo e/ou possíveis discrepâncias existentes entre eles. Inicialmente, comparou-se as coordenadas absolutas de cada um dos vértices isoladamente, o que é possível uma vez que as coordenadas para o ponto origem, tanto no STL quanto no SGL, são as mesmas $\left(X_{0}=e_{o}=150000,000 \mathrm{~m} ; Y_{o}=n_{o}=250000,000 \mathrm{~m} \mathrm{e} Z_{0}=u_{o}\right)$. 
Como ponto origem dos dois sistemas em análise, adotou-se um ponto virtual, denominado de ORIGEM, cujas coordenadas geodésicas correspondem à média da latitude e da longitude dos vinte e nove pontos em análise, adotandose para a altitude a média das altitudes ortométricas dos mesmos pontos, conforme recomendação do Manual Técnico de Posicionamento, para o Sistema Geodésico Local, e da NBR 14166/98, para o Sistema Topográfico Local.

A segunda análise realizada se deu a partir de grandezas lineares, calculandose o perímetro $(P)$ do polígono formado a partir dos vinte e oito marcos, que consiste na somatória do comprimento dos lados de um polígono (Equação 28). Para determinar a distância entre dois marcos consecutivos, recorreu-se duas metodologias distintas:

a) 1ạ: Cálculo da distância euclidiana (d) a partir das coordenadas tridimensionais conhecidas de dois pontos, conforme a Equação 29, apresentada em Venturi (1949), utilizando-se as coordenadas topográficas locais $(X, Y, Z)$ ou as coordenadas cartesianas locais $(e, n, u)$;

b) 2ạ: Cálculo da distância topocêntrica $(d t)$ levando-se em consideração a altitude elipsoidal, conforme a Equação 30, recomendada por INCRA (2013a).

$$
\begin{aligned}
& P=\sum_{i=1}^{l} d_{i} \\
& d=\sqrt{\left(x_{2}-x_{1}\right)^{2}+\left(y_{2}-y_{1}\right)^{2}+\left(z_{2}-z_{1}\right)^{2}} \\
& d t=\left[\left(X_{c_{A}}-X_{c_{b}}\right)^{2}+\left(Y_{c_{A}}-Y_{c_{B}}\right)^{2}+\left(Z_{c_{A}}-Z_{c_{B}}\right)^{2}-\left(h_{A}-h_{B}\right)^{2}\right]^{1 / 2}
\end{aligned}
$$

Sendo:

$d_{i}=$ distância entre dois vértices consecutivos;

$l=$ total de lados do polígono;

$x_{1}, y_{1}, z_{1}=$ coordenadas tridimensionais de um ponto, correspondentes a $X_{1}, Y_{1}, Z_{1}$ ou a $e_{1}, n_{1}, u_{1}$, conforme o caso;

$x_{2}, y_{2}, z_{2}=$ coordenadas tridimensionais do ponto consecutivo, correspondentes a $X_{2}, Y_{2}, Z_{2}$ ou a $e_{2}, n_{2}, u_{2}$, conforme o caso;

$X_{c}, Y_{c}, Z_{c}=$ coordenadas cartesianas geocêntricas;

$h=$ altitude elipsoidal.

Obteve-se, assim, três conjuntos de distâncias (distâncias horizontais a partir das coordenadas topográficas locais, distâncias topocêntricas a partir das coordenadas geodésicas locais e distâncias também topocêntricas a partir das coordenadas cartesianas geocêntricas), calculando-se três perímetros distintos.

Por fim, calculou-se a área do polígono formado pelos marcos considerados através das coordenadas locais nos dois sistemas analisados. De acordo com Veiga, Zanetti e Faggion (2012), o cálculo da área $(A)$ pode ser realizado a partir do cálculo da área de trapézios formados pelos vértices da poligonal, que consiste na Fórmula de Gauss, apresentada pela Equação 31. 
Em que:

$x, y=$ coordenadas retangulares dos vértices do polígono, correspondentes a $X, Y$ ou a $e, n$, conforme o caso;

$t=$ total de vértices do polígono considerado. Para o estudo em questão, $\mathrm{t}$ equivale à quantidade de marcos rastreados $(t=28)$. Deve-se observar que quando $\mathrm{i}=\mathrm{t}$, o valor de $\mathrm{i}+1$ deve ser considerado como sendo 1 , ou seja, 0 primeiro ponto novamente (VEIGA; ZANETTI; FAGGION, 2012).

\section{RESULTADOS E DISCUSSÃO}

Na Tabela 1 apresenta-se as coordenadas geodésicas, determinadas a partir do posicionamento por GNSS e processamento dos dados, dos vinte e oito marcos considerados, da estação geodésica 93949 e do ponto virtual utilizado como origem, bem como as ondulações geoidais (G), calculadas a partir do software MAPGEO 2015 v1.0, e as referidas altitudes ortométricas (H) para os trinta vértices em questão.

Tabela 1 - Coordenadas geodésicas dos vinte e oito marcos considerados, da estação homologada pelo IBGE (93949) e do ponto ORIGEM, no sistema de referência SIRGAS2000

\begin{tabular}{|c|c|c|c|c|c|}
\hline Vértice & $\varphi$ & $\lambda$ & $h(m)$ & $G(m)$ & $H(m)$ \\
\hline 93949 & $22^{\circ} 19^{\prime} 09,768700^{\prime \prime S}$ & $46^{\circ} 19^{\prime} 44,113998^{\prime \prime} \mathrm{W}$ & 884,825 & $-2,76$ & 887,585 \\
\hline M1 & $22^{\circ} 18^{\prime} 50,370570$ 'S & $46^{\circ} 19^{\prime} 26,099830^{\prime \prime} \mathrm{W}$ & 848,394 & $-2,75$ & 851,144 \\
\hline $\mathrm{M} 2$ & $22^{\circ} 18^{\prime} 48,472340^{\prime \prime S}$ & $46^{\circ} 19^{\prime} 25,893410^{\prime \prime} \mathrm{W}$ & 849,017 & $-2,75$ & 851,767 \\
\hline M3 & $22^{\circ} 18^{\prime} 44,712580^{\prime \prime S}$ & $46^{\circ} 19^{\prime} 25,835070^{\prime \prime} \mathrm{W}$ & 862,299 & $-2,75$ & 865,049 \\
\hline M4 & $22^{\circ} 18^{\prime} 41,628560^{\prime \prime S}$ & $46^{\circ} 19^{\prime} 25,831420^{\prime \prime} \mathrm{W}$ & 879,909 & $-2,76$ & 882,669 \\
\hline M5 & $22^{\circ} 18^{\prime} 36,413820^{\prime \prime S}$ & $46^{\circ} 19^{\prime} 25,785940^{\prime \prime} \mathrm{W}$ & 904,668 & $-2,76$ & 907,428 \\
\hline M6 & $22^{\circ} 18^{\prime} 32,085010^{\prime \prime} \mathrm{S}$ & $46^{\circ} 19^{\prime} 25,682240^{\prime \prime} \mathrm{W}$ & 895,847 & $-2,76$ & 898,607 \\
\hline M7 & $22^{\circ} 18^{\prime} 28,579520^{\prime \prime} \mathrm{S}$ & $46^{\circ} 19^{\prime} 25,613680^{\prime \prime} \mathrm{W}$ & 906,162 & $-2,76$ & 908,922 \\
\hline M8 & $22^{\circ} 18^{\prime} 24,886720^{\prime \prime S}$ & $46^{\circ} 19^{\prime} 25,702600^{\prime \prime W}$ & 899,686 & $-2,76$ & 902,446 \\
\hline M9 & $22^{\circ} 18^{\prime} 20,875660^{\prime \prime S}$ & $46^{\circ} 19^{\prime} 25,688710^{\prime \prime} \mathrm{W}$ & 885,301 & $-2,76$ & 888,061 \\
\hline M10 & $22^{\circ} 18^{\prime} 20,797620^{\prime \prime S}$ & $46^{\circ} 19^{\prime} 31,787980^{\prime \prime} \mathrm{W}$ & 927,226 & $-2,77$ & 929,996 \\
\hline M11 & $22^{\circ} 18^{\prime} 20,912930^{\prime \prime S}$ & $46^{\circ} 19^{\prime} 37,695730^{\prime \prime} \mathrm{W}$ & 897,254 & $-2,78$ & 900,034 \\
\hline M12 & $22^{\circ} 18^{\prime} 20,817410^{\prime \prime S}$ & $46^{\circ} 19^{\prime} 42,774050^{\prime \prime} \mathrm{W}$ & 880,004 & $-2,78$ & 882,784 \\
\hline M13 & $22^{\circ} 18^{\prime} 20,699480^{\prime \prime S}$ & $46^{\circ} 19^{\prime} 51,405300^{\prime \prime} \mathrm{W}$ & 939,646 & $-2,79$ & 942,436 \\
\hline M14 & $22^{\circ} 18^{\prime} 17,173330^{\prime \prime} \mathrm{S}$ & $46^{\circ} 19^{\prime} 51,358960^{\prime \prime} \mathrm{W}$ & 930,788 & $-2,79$ & 933,578 \\
\hline M15 & $22^{\circ} 18^{\prime} 12,522930^{\prime \prime S}$ & $46^{\circ} 19^{\prime} 51,325140^{\prime \prime} \mathrm{W}$ & 908,971 & $-2,79$ & 911,761 \\
\hline M16 & $22^{\circ} 18^{\prime} 08,434280^{\prime \prime S}$ & $46^{\circ} 19^{\prime} 51,298370^{\prime \prime} \mathrm{W}$ & 894,331 & $-2,80$ & 897,131 \\
\hline M17 & $22^{\circ} 18^{\prime} 04,291660^{\prime \prime S}$ & $46^{\circ} 19^{\prime} 51,122400^{\prime \prime} \mathrm{W}$ & 886,486 & $-2,80$ & 889,286 \\
\hline M18 & $22^{\circ} 18^{\prime} 05,761800^{\prime \prime S}$ & $46^{\circ} 20^{\prime} 14,866440^{\prime \prime} \mathrm{W}$ & 876,742 & $-2,82$ & 879,562 \\
\hline
\end{tabular}




\begin{tabular}{cccccc}
\hline M19 & $22^{\circ} 18^{\prime} 10,433560^{\prime \prime S}$ & $46^{\circ} 20^{\prime} 15,285210^{\prime \prime} \mathrm{W}$ & 883,206 & $-2,82$ & 886,026 \\
\hline $\mathrm{M} 20$ & $22^{\circ} 18^{\prime} 16,565890^{\prime \prime} \mathrm{S}$ & $46^{\circ} 20^{\prime} 15,526660^{\prime \prime} \mathrm{W}$ & 889,984 & $-2,82$ & 892,804 \\
\hline $\mathrm{M} 21$ & $22^{\circ} 18^{\prime} 26,061590^{\prime \prime} \mathrm{S}$ & $46^{\circ} 20^{\prime} 15,363530^{\prime \prime} \mathrm{W}$ & 927,961 & $-2,81$ & 930,771 \\
\hline $\mathrm{M} 22$ & $22^{\circ} 18^{\prime} 30,267830^{\prime \prime} \mathrm{S}$ & $46^{\circ} 20^{\prime} 15,430690^{\prime \prime} \mathrm{W}$ & 936,086 & $-2,81$ & 938,896 \\
\hline $\mathrm{M} 23$ & $22^{\circ} 18^{\prime} 38,087560^{\prime \prime} \mathrm{S}$ & $46^{\circ} 20^{\prime} 15,564450^{\prime \prime} \mathrm{W}$ & 926,552 & $-2,81$ & 929,362 \\
\hline $\mathrm{M} 24$ & $22^{\circ} 18^{\prime} 41,373920^{\prime \prime} \mathrm{S}$ & $46^{\circ} 20^{\prime} 15,742570^{\prime \prime} \mathrm{W}$ & 892,771 & $-2,81$ & 895,581 \\
\hline $\mathrm{M} 25$ & $22^{\circ} 18^{\prime} 47,333320^{\prime \prime} \mathrm{S}$ & $46^{\circ} 20^{\prime} 15,831210^{\prime \prime} \mathrm{W}$ & 887,578 & $-2,80$ & 890,378 \\
\hline $\mathrm{M} 26$ & $22^{\circ} 18^{\prime} 52,810370^{\prime \prime} \mathrm{S}$ & $46^{\circ} 20^{\prime} 15,946990^{\prime \prime} \mathrm{W}$ & 892,585 & $-2,80$ & 895,385 \\
\hline $\mathrm{M} 27$ & $22^{\circ} 18^{\prime} 56,463250^{\prime \prime} \mathrm{S}$ & $46^{\circ} 20^{\prime} 16,025500^{\prime \prime} \mathrm{W}$ & 868,047 & $-2,80$ & 870,847 \\
\hline $\mathrm{M} 28$ & $22^{\circ} 18^{\prime} 59,575230^{\prime \prime} \mathrm{S}$ & $46^{\circ} 20^{\prime} 15,892890^{\prime \prime} \mathrm{W}$ & 847,279 & $-2,80$ & 850,079 \\
\hline ORIGEM & $22^{\circ} 18^{\prime} 31,320000^{\prime \prime} \mathrm{S}$ & $46^{\circ} 19^{\prime} 50,910000^{\prime \prime} \mathrm{W}$ & 893,435 & $-2,79$ & 896,220 \\
\hline
\end{tabular}

Fonte: Autoria própria (2016).

De posse das coordenadas geodésicas, obteve-se as coordenadas geocêntricas para os trinta vértices, conforme a Tabela 2, e, a partir dessas, calculou-se as coordenadas cartesianas locais, referidas ao SGL e acrescidas dos valores iniciais $e_{0}=150000 \mathrm{~m}, \mathrm{n}_{0}=250000 \mathrm{~m}$ e $\mathrm{u}_{0}=896,220 \mathrm{~m}$. A Tabela 3 apresenta as coordenadas cartesianas locais (e, n, u) e as coordenadas topográficas locais $(X, Y, Z)$, também determinas para os trinta vértices.

Tabela 2 - Coordenadas geocêntricas dos trinta vértices em consideração

\begin{tabular}{|c|c|c|c|}
\hline Vértice & $X c(m)$ & Yc (m) & $\mathrm{Zc}(\mathrm{m})$ \\
\hline 93949 & 4076790,081 & $-4270430,569$ & $-2407502,380$ \\
\hline M1 & 4077296,239 & $-4270214,024$ & $-2406936,483$ \\
\hline M2 & 4077316,222 & $-4270226,396$ & $-2406882,696$ \\
\hline M3 & 4077356,241 & $-4270265,891$ & $-2406780,735$ \\
\hline M4 & 4077392,442 & $-4270303,653$ & $-2406699,648$ \\
\hline M5 & 4077451,260 & $-4270363,368$ & $-2406560,631$ \\
\hline M6 & 4077482,682 & $-4270391,979$ & $-2406434,080$ \\
\hline M7 & 4077518,962 & $-4270427,133$ & $-2406338,224$ \\
\hline M8 & 4077542,763 & $-4270455,745$ & $-2406230,663$ \\
\hline M9 & 4077566,204 & $-4270479,720$ & $-2406111,041$ \\
\hline M10 & 4077467,338 & $-4270629,005$ & $-2406124,733$ \\
\hline M11 & 4077324,941 & $-4270724,758$ & $-2406116,639$ \\
\hline M12 & 4077209,543 & $-4270814,406$ & $-2406107,373$ \\
\hline M13 & 4077069,876 & $-4271025,924$ & $-2406126,653$ \\
\hline M14 & 4077093,606 & $-4271048,861$ & $-2406022,930$ \\
\hline M15 & 4077117,860 & $-4271072,867$ & $-2405882,288$ \\
\hline M16 & 4077142,023 & $-4271097,069$ & $-2405760,359$ \\
\hline M17 & 4077174,049 & $-4271123,324$ & $-2405639,472$ \\
\hline M18 & 4076664,281 & $-4271573,699$ & $-2405677,619$ \\
\hline M19 & 4076622,082 & $-4271546,846$ & $-2405813,042$ \\
\hline M20 & 4076571,980 & $-4271504,359$ & $-2405990,154$ \\
\hline M21 & 4076523,065 & $-4271446,342$ & $-2406274,834$ \\
\hline M22 & 4076492,953 & $-4271417,574$ & $-2406397,635$ \\
\hline
\end{tabular}




\begin{tabular}{clll}
\hline M23 & 4076421,044 & $-4271347,773$ & $-2406616,575$ \\
\hline M24 & 4076369,280 & $-4271300,919$ & $-2406697,284$ \\
\hline M25 & 4076316,073 & $-4271248,842$ & $-2406864,920$ \\
\hline M26 & 4076272,705 & $-4271208,201$ & $-2407022,699$ \\
\hline M27 & 4076225,947 & $-4271162,462$ & $-2407117,343$ \\
\hline M28 & 4076190,331 & $-4271119,644$ & $-2407198,023$ \\
\hline ORIGEM & 4076964,935 & $-4270895,601$ & $-2406411,290$ \\
\hline
\end{tabular}

Fonte: Autoria própria (2016).

Tabela 3 - Coordenadas Cartesianas Locais (e, n, u), referidas ao SGL, e Coordenadas Topográficas Locais (X, Y, Z), referidas ao STL, para os vinte e oito marcos em estudo, para a estação 93949 e para o ponto origem

\begin{tabular}{|c|c|c|c|c|c|c|}
\hline Vértice & e $(m)$ & $n(m)$ & $\mathrm{u}(\mathrm{m})$ & $X(m)$ & $Y(m)$ & $Z(m)$ \\
\hline 93949 & 150194,623 & 248817,059 & 887,497 & 150194,524 & 248817,166 & 887,585 \\
\hline M1 & 150710,270 & 249413,809 & 851,113 & 150710,176 & 249413,914 & 851,144 \\
\hline $\mathrm{M} 2$ & 150716,181 & 249472,205 & 851,740 & 150716,087 & 249472,310 & 851,767 \\
\hline M3 & 150717,858 & 249587,869 & 865,030 & 150717,763 & 249587,975 & 865,049 \\
\hline M4 & 150717,969 & 249682,744 & 882,646 & 150717,871 & 249682,852 & 882,669 \\
\hline M5 & 150719,281 & 249843,168 & 907,411 & 150719,181 & 249843,278 & 907,428 \\
\hline M6 & 150722,255 & 249976,340 & 898,591 & 150722,155 & 249976,449 & 898,607 \\
\hline M7 & 150724,223 & 250084,182 & 908,906 & 150724,123 & 250084,291 & 908,922 \\
\hline M8 & 150721,683 & 250197,787 & 902,427 & 150721,583 & 250197,896 & 902,446 \\
\hline M9 & 150722,084 & 250321,183 & 888,037 & 150721,986 & 250321,292 & 888,061 \\
\hline M10 & 150547,490 & 250323,593 & 929,979 & 150547,388 & 250323,700 & 929,996 \\
\hline M11 & 150378,372 & 250320,049 & 900,020 & 150378,272 & 250320,158 & 900,034 \\
\hline M12 & 150232,999 & 250322,989 & 882,777 & 150232,900 & 250323,099 & 882,784 \\
\hline M13 & 149985,921 & 25032 & 942,423 & 149985,822 & 250326,729 & 942,436 \\
\hline M14 & 149987,248 & 250435,100 & 933,558 & 149987,148 & 250435,207 & 933,578 \\
\hline M15 & 149988,216 & 250578,164 & 911,730 & 149988,116 & 250578,271 & 911,761 \\
\hline M16 & 149988,982 & 250703,945 & 897,077 & 149988,882 & 250704,054 & 897,131 \\
\hline M17 & 149994,019 & 250831,387 & 889,217 & 149993,920 & 250831,497 & 889,286 \\
\hline M18 & 149314,303 & 250786,144 & 879,442 & 149314,201 & 250786,255 & 879,562 \\
\hline M19 & 149302,321 & 250642,423 & 885,921 & 149302,219 & 250642,533 & 886,026 \\
\hline M20 & 149295,417 & 250453,769 & 892,714 & 149295,316 & 250453,878 & 892,804 \\
\hline M21 & 149300,095 & 250161,646 & 930,706 & 149299,999 & 250161,754 & 930,771 \\
\hline M22 & 149298,178 & 250032,244 & 938,833 & 149298,082 & 250032,353 & 938,896 \\
\hline M23 & 149294,361 & 249791,678 & 929,295 & 149294,264 & 249791,787 & 929,362 \\
\hline M24 & 149289,271 & 249690,577 & 895,509 & 149289,170 & 249690,686 & 895,581 \\
\hline M25 & 149286,742 & 249507,243 & 890,304 & 149286,641 & 249507,351 & 890,378 \\
\hline M26 & 149283,435 & 249338,747 & 895,295 & 149283,335 & 249338,856 & 895,385 \\
\hline M27 & 149281,196 & 249226,373 & 870,745 & 149281,093 & 249226,479 & 870,847 \\
\hline M28 & 149284,999 & 249130,639 & 849,965 & 149284,893 & 249130,742 & 850,079 \\
\hline ORIGEM & 150000,000 & 250000,000 & 896,220 & 150000,000 & 250000,000 & 896,220 \\
\hline
\end{tabular}

Fonte: Autoria própria (2016). 
Realizando-se a análise pontual a partir dos resultados apresentados na Tabela 3, destaca-se, na Tabela 4 , as discrepâncias $(\Delta x, \Delta y$ e $\Delta z)$ entre as coordenadas cartesianas locais e as topográficas locais para cada um dos vértices em estudo. Analisando-se tais valores, observa-se que as diferenças existentes entre os dois sistemas em estudo são pequenas, porém consideráveis, uma vez que os erros médios em $x, y$ e $z$, calculados a partir da média das diferenças $\Delta x$, $\Delta y$ e $\Delta z$ encontradas para os trinta vértices, giram em torno de $9,6 \mathrm{~cm}$ para o eixo $x, 10,4 \mathrm{~cm}$ para o eixo y e $4,9 \mathrm{~cm}$ para o eixo $z$.

Calculando-se o Erro Posicional Tridimensional (EPT), também apresentado na Tabela 4, para a estação geodésica e para os vinte e oito marcos em estudo, a partir das diferenças pontuais tridimensionais ( $\Delta x, \Delta y$ e $\Delta z$ ) entre os dois sistemas analisados, observa-se uma diferença média de $15 \mathrm{~cm}$ entre as coordenadas topográficas locais e as cartesianas locais para os vinte e nove vértices, bem como constata-se que os maiores valores de EPT são obtidos para os vértices mais distantes do vértice ORIGEM. Ainda na Tabela 4, observa-se que o Erro Planimétrico (EP), calculado para os vinte e nove vértices a partir das diferenças em $\mathrm{x}$ e y $(\Delta \mathrm{x}$ e $\Delta \mathrm{y})$ entre os sistemas analisados, não é influenciado pela distância do ponto à origem, sendo que o mesmo varia entre 14 e $16 \mathrm{~cm}$ para todos os vértices analisados.

Tabela 4 - Diferenças ( $\Delta \mathrm{x}, \Delta \mathrm{y}$ e $\Delta \mathrm{z}$ ) encontradas entre as coordenadas cartesianas locais $(e, n, u)$ e as coordenadas topográficas locais $(X, Y, Z)$ para os trinta vértices em estudo e DO (Distância à origem), EP e EPT, calculados a partir das referidas diferenças, para os marcos analisados e para a estação 93949

\begin{tabular}{|c|c|c|c|c|c|c|}
\hline Vértice & $\Delta x(m)$ & $\Delta y(m)$ & $\Delta z(m)$ & $\mathrm{EP}(\mathrm{m})$ & EPT $(m)$ & DO (m) \\
\hline 93949 & 0,099 & 0,107 & 0,088 & 0,146 & 0,171 & 1198,723 \\
\hline M1 & 0,094 & 0,105 & 0,031 & 0,141 & 0,144 & 920,786 \\
\hline M2 & 0,094 & 0,105 & 0,027 & 0,141 & 0,144 & 889,515 \\
\hline M3 & 0,096 & 0,107 & 0,019 & 0,143 & 0,145 & 827,615 \\
\hline M4 & 0,098 & 0,108 & 0,023 & 0,146 & 0,147 & 784,807 \\
\hline M5 & 0,100 & 0,109 & 0,017 & 0,148 & 0,149 & 736,059 \\
\hline M6 & 0,099 & 0,109 & 0,016 & 0,147 & 0,148 & 722,539 \\
\hline M7 & 0,100 & 0,109 & 0,016 & 0,148 & 0,149 & 729,012 \\
\hline M8 & 0,100 & 0,109 & 0,019 & 0,147 & 0,149 & 748,228 \\
\hline M9 & 0,098 & 0,109 & 0,024 & 0,147 & 0,149 & 790,248 \\
\hline M10 & 0,102 & 0,107 & 0,017 & 0,148 & 0,149 & 635,937 \\
\hline M11 & 0,100 & 0,109 & 0,014 & 0,147 & 0,148 & 495,571 \\
\hline M12 & 0,099 & 0,110 & 0,007 & 0,148 & 0,148 & 398,291 \\
\hline M13 & 0,099 & 0,106 & 0,013 & 0,146 & 0,146 & 327,036 \\
\hline M14 & 0,099 & 0,106 & 0,020 & 0,146 & 0,147 & 435,396 \\
\hline M15 & 0,100 & 0,107 & 0,031 & 0,146 & 0,150 & 578,393 \\
\hline M16 & 0,100 & 0,109 & 0,054 & 0,147 & 0,157 & 704,142 \\
\hline M17 & 0,100 & 0,110 & 0,069 & 0,148 & 0,164 & 831,519 \\
\hline M18 & 0,102 & 0,111 & 0,120 & 0,151 & 0,193 & 1043,320 \\
\hline M19 & 0,101 & 0,110 & 0,105 & 0,150 & 0,183 & 948,549 \\
\hline M20 & 0,101 & 0,109 & 0,090 & 0,148 & 0,174 & 838,203 \\
\hline
\end{tabular}




\begin{tabular}{ccccccc}
\hline M21 & 0,096 & 0,108 & 0,065 & 0,145 & 0,159 & 718,447 \\
\hline M22 & 0,096 & 0,109 & 0,063 & 0,145 & 0,158 & 702,663 \\
\hline M23 & 0,097 & 0,110 & 0,067 & 0,146 & 0,161 & 735,809 \\
\hline M24 & 0,100 & 0,109 & 0,072 & 0,148 & 0,165 & 775,212 \\
\hline M25 & 0,101 & 0,108 & 0,074 & 0,148 & 0,165 & 866,939 \\
\hline M26 & 0,100 & 0,109 & 0,090 & 0,148 & 0,173 & 975,049 \\
\hline M27 & 0,103 & 0,106 & 0,102 & 0,148 & 0,180 & 1056,013 \\
\hline M28 & 0,105 & 0,102 & 0,114 & 0,147 & 0,186 & 1125,605 \\
\hline ORIGEM & 0,000 & 0,000 & 0,000 & - & - & - \\
\hline
\end{tabular}

Fonte: Autoria própria (2016).

Pela Tabela 4, percebe-se que os valores de EP são menores que os valores de EPT, o que aponta que a altitude interfere nas discrepâncias encontradas entre os sistemas estudados. Um dos diversos fatores que influenciam na altitude dos vértices é o fato de que o software MAPGEO 2015 v1.0 fornece valores interpolados para a ondulação geoidal dos pontos. Soma-se a isso, o fato de que, para o SGL, considera-se um plano tangente à superfície terrestre no ponto origem do sistema e que é perpendicular a normal ao elipsoide, o qual possui valor correspondente à altitude ortométrica média dos pontos em questão, e, consequentemente, todos os demais valores encontrados para o eixo u, estão relacionadas a esse plano tangente; enquanto que, para o STL, considerou-se para o eixo z os valores correspondentes às altitudes ortométricas dos referidos pontos, as quais são determinadas a partir do geoide, uma vez que, para esse sistema, adota-se uma superfície esférica denominada de superfície de nível médio do terreno, que é projetada sem deformação no Plano Topográfico Local.

A segunda análise realizada, apresentada na Tabela 5 , se deu a partir de grandezas lineares, calculando-se três conjuntos de distâncias e, portanto, três valores de perímetro.

Tabela 5 - Distâncias calculadas entre marcos consecutivos a partir das coordenadas cartesianas locais (Cart. Locais), topográficas locais (Topo. Locais) e geocêntricas, bem como os respectivos perímetros obtidos pela somatória de cada conjunto de distância

\section{Distância Cart. Locais $(m) \quad$ Topo. Locais $(m) \quad$ Geocêntricas $(m)$}

\begin{tabular}{cccc}
\hline$d_{1-2}$ & 58,694 & 58,695 & 58,695 \\
\hline$d_{2-3}$ & 115,676 & 115,677 & 115,676 \\
\hline$d_{3-4}$ & 94,875 & 94,877 & 94,876 \\
\hline$d_{4-5}$ & 160,429 & 160,431 & 160,431 \\
\hline$d_{5-6}$ & 133,205 & 133,204 & 133,204 \\
\hline$d_{6-7}$ & 107,860 & 107,860 & 107,861 \\
\hline$d_{7-8}$ & 113,633 & 113,633 & 113,634 \\
\hline$d_{8-9}$ & 123,397 & 123,396 & 123,396 \\
\hline$d_{9-10}$ & 174,611 & 174,615 & 174,615 \\
\hline$d_{10-11}$ & 169,155 & 169,153 & 169,153 \\
\hline$d_{11-12}$ & 145,403 & 145,402 & 145,402 \\
\hline$d_{12-13}$ & 247,105 & 247,105 & 247,106 \\
\hline$d_{13-14}$ & 108,486 & 108,486 & 108,487 \\
\hline
\end{tabular}




\begin{tabular}{cccc}
\hline$d_{14-15}$ & 143,067 & 143,068 & 143,068 \\
\hline$d_{15-16}$ & 125,783 & 125,785 & 125,785 \\
\hline$d_{16-17}$ & 127,542 & 127,543 & 127,543 \\
\hline$d_{17-18}$ & 681,220 & 681,223 & 681,221 \\
\hline$d_{18-19}$ & 144,220 & 144,221 & 144,220 \\
\hline$d_{19-20}$ & 188,780 & 188,781 & 188,781 \\
\hline$d_{20-21}$ & 292,160 & 292,162 & 292,163 \\
\hline$d_{21-22}$ & 129,416 & 129,415 & 129,415 \\
\hline$d_{22-23}$ & 240,596 & 240,596 & 240,597 \\
\hline$d_{23-24}$ & 101,229 & 101,230 & 101,230 \\
\hline$d_{24-25}$ & 183,351 & 183,352 & 183,352 \\
\hline$d_{25-26}$ & 168,528 & 168,528 & 168,528 \\
\hline$d_{26-27}$ & 112,396 & 112,399 & 112,399 \\
\hline$d_{27-28}$ & 95,810 & 95,812 & 95,811 \\
\hline$d_{28-1}$ & 1453,129 & 1453,141 & 1453,129 \\
\hline Perímetro & $\mathbf{5 9 3 9 , 7 5 8}$ & $\mathbf{5 9 3 9 , 7 8 9}$ & $\mathbf{5 9 3 9 , 7 7 9}$ \\
\hline
\end{tabular}

Fonte: Autoria própria (2016).

Pelos valores de perímetro obtidos, vê-se que a diferença linear entre o STL e o SGL é de 3,1 cm para a área em estudo, uma diferença pequena, mas que não pode ser desconsiderada para trabalhos de alta precisão. Salienta-se que a fórmula para cálculo da distância topocêntrica utilizando as coordenadas geocêntricas e levando em consideração as alturas geométricas dos vértices em questão, conforme é recomendado pelo INCRA (2013a), é válida e confiável, apresentando valores lineares próximos aos obtidos pela equação da distância euclidiana, sendo que o perímetro calculado a partir das coordenadas geocêntricas difere-se do perímetro dado pelas coordenadas cartesianas locais em $2,1 \mathrm{~cm}$, e em $1 \mathrm{~cm}$ do perímetro obtido a partir das coordenadas topográficas locais, considerando-se as aproximações efetuadas com três casas decimais.

Por fim, analisou-se a diferença entre a área obtida a partir das coordenadas topográficas locais e a partir das coordenadas cartesianas locais. Conforme a Tabela 6, nota-se que a diferença entre as duas áreas obtidas é de $8,736 \mathrm{~m}^{2}$. Expressando-se a área em hectares, conforme exigência do INCRA (2013a), é observada uma diferença de 0,0009 hectares entre as áreas obtidas, o que contribui para comprovar a pequena discrepância entre os sistemas estudados.

Tabela 6 - Valores de área, em metros quadrados $\left(\mathrm{m}^{2}\right)$ e em hectares (ha), calculados a partir das Coordenadas Topográficas Locais e das Coordenadas Cartesianas Locais

\begin{tabular}{ccc} 
Coordenadas & $\mathbf{A}\left(\mathbf{m}^{\mathbf{2}}\right)$ & A (ha) \\
\hline Topográficas Locais & 1832294,664 & 183,2295 \\
\hline Cartesianas Locais & 1832285,928 & 183,2286 \\
\hline
\end{tabular}

Fonte: Autoria própria (2016). 


\section{CONCLUSÕES}

Ao término do presente estudo, conclui-se que os dois sistemas analisados, Sistema Topográfico Local e Sistema Geodésico Local, não são compatíveis, uma vez que as análises realizadas a partir das grandezas pontuais, lineares e de superfície nos dois sistemas em estudo, apontam para uma divergência considerável quando na adoção dos sistemas para as aplicações descritas na NBR 14166/98 e no Manual Técnico de Posicionamento (INCRA, 2013a). Destaca-se que as diferenças lineares e de superfície entre os dois sistemas são significativas, o que contribui para a conclusão de que ambos os sistemas não são equivalentes e, desse modo, cada qual deve ser utilizado para uma finalidade, conforme recomendam as normas respectivas a cada um.

Pelas análises efetuadas, os vértices mais afastados da origem dos sistemas apresentam maior EPT. Afirma-se que fatores decorrentes da curvatura terrestre, do desvio da vertical e do próprio campo de gravidade terrestre interferem na determinação das altitudes ortométricas à medida que se afasta do ponto origem. Diante do exposto, recomenda-se a realização de trabalhos futuros nos quais seja realizada uma nova comparação das coordenadas obtidas nos dois sistemas de coordenadas locais, considerando-se vértices mais distantes do ponto origem e mais próximos às extremidades do Plano Topográfico Local, isto é, que distem cerca de $50 \mathrm{Km}$ do ponto Origem, a fim de verificar a interferência da distância nos resultados obtidos, principalmente no que diz respeito ao EPT.

Ressalta-se, ainda, que embora a diferença observada entre as distâncias calculadas (distâncias euclidianas e a distância topocêntrica) seja pequena, não é desprezível, e, portanto, o recomendável em trabalhos de georreferenciamento de imóveis rurais, conforme o Manual Técnico de Posicionamento do INCRA, é a utilização da Equação 30 quando no cálculo do perímetro.

Por fim, considerando-se a aplicabilidade dos Sistemas Geodésico Local e Topográfico Local, conforme o Manual Técnico de Posicionamento do INCRA e a NBR 14166/98, bem como as análises efetuadas que demonstram uma pequena, mas considerável diferença entre os dois sistemas de coordenadas em estudo, recomenda-se que, para trabalhos práticos de georreferenciamento de imóveis rurais, adote-se o Sistema Geodésico Local, e, em levantamentos cadastrais municipais, recomenda-se a adoção do Sistema Topográfico Local, conforme exigência da NBR 14166/98 quando na implantação da Rede de Referência Cadastral, assumindo-se, para o eixo $z$, as próprias altitudes ortométricas dos vértices em questão, o que resulta em dados altimétricos mais confiáveis. 


\title{
Comparative analysis of the coordinates in the Local Geodetic System and in the Local Topographic System
}

\begin{abstract}
One of the important activities of professionals in Geodesy consists of the transformations between coordinate systems and, depending on the purpose, one should resort to one or another system. The local coordinate systems provide greater precision in the implementation of engineering works and cadastral works, among which stands out the Local Topographical System, adopted in municipal surveys for registration purposes, according to NBR 14166/98, and the Local Geodetic System applied when the georeferencing of rural properties, according to the Positioning of Technical Manual of NTGIR (INCRA, 2013a). In order to perform a comparative analysis of the obtained coordinates in the three-dimensional local coordinate systems, is adopted as an object of study, twenty-eight marks located on IFSULDEMINAS and one geodetic stations approved by the IBGE, which had their geodetic coordinates obtained by positioning by GNSS, with static and static fast methods. Conclude, from the obtained local coordinates and the specific, linear and surface comparisons realized, that both systems have a small divergence however significant, which means that the same are not considered equivalent and, therefore, the applicability of each one must comply with the requirements of technical standards.
\end{abstract}

KEYWORDS: GNSS. Geodetic System. Topographic System. Local coordinates. 


\section{REFERÊNCIAS}

ASSOCIAÇÃO BRASILEIRA DE NORMAS TÉCNICAS. NBR 14166: Rede de Referência Cadastral Municipal - Procedimento. Rio de Janeiro, 1998. 23 p.

HOFMANN-WELLENHOF, B.; LICHTENEGGER, H.; WASLE, E. GNSS - Global Navigation Satellite Systems, GPS, GLONASS, Galileo and more. Springer-Verlag Wien, 2008. 501p.

IBGE. Instituto Brasileiro de Geografia e Estatística. Sistema Geodésico Brasileiro: Banco de Dados Geodésicos. Disponível em: <http://www.ibge.gov.br/home/geociencias/geodesia/bdgpesq_googlemaps.php \#tabela_dados>. Acesso em: 27 abr. 2016.

INSTITUTO NACIONAL DE COLONIZAÇÃO E REFORMA AGRÁRIA. Manual Técnico de Posicionamento: Georreferenciamento de Imóveis Rurais. 1 ed. Brasília, 2013a. 34 p.

INSTITUTO NACIONAL DE COLONIZAÇÃO E REFORMA AGRÁRIA. Norma Técnica para Georreferenciamento de Imóveis Rurais. 3 ed. Brasília, 2013b. 4 p.

MONICO, J.F.G. Posicionamento pelo GNSS: descrição, fundamentos e aplicações. 2. ed. São Paulo: Editora UNESP, 2008. 477p.

RAPP, R. H. Geometric Geodesy Part I. Columbus, Ohio: The Ohio State University, 1991. $178 \mathrm{p}$.

VEIGA, L. A. K.; ZANETTI, M. A. Z.; FAGGION, P. L. Fundamentos de Topografia. Paraná: UFPR - Universidade Federal do Paraná, 2012. 274 p.

VENTURI, J. J. Álgebra Vetorial e Geometria Analítica. 9. ed. Curitiba: UFPR, 1949. $242 \mathrm{p}$. 
Recebido: 12 ago. 2016

Aprovado: 21 mar. 2017

DOI: $10.3895 /$ rbgeo.v5n1.5420

Como citar: SIMÕES, D. P.; ALBARICI, F. L.; BORGES, P. A. F. Análise comparativa das coordenadas no

Sistema Geodésico Local e no Sistema Topográfico Local. R. bras. Geom., Curitiba, v. 5, n. 1, p. 062-081,

jan/mar. 2017. Disponível em: <https://periodicos.utfpr.edu.br/rbgeo>. Acesso em: XXX.

Correspondência:

Débora Paula Simões

Sítio Real, s/n, Bairro Guabiroba, CEP 37578-000, Bueno Brandão, Minas Gerais, Brasil

Direito autoral: Este artigo está licenciado sob os termos da Licença Creative Commons-Atribuição 4.0

Internacional.

(c) (1) 